\title{
Neurodevelopment is Particular Vulnerable in Neonatal Male Rats Subjected to Maternal Separation
}

\author{
Chunyao Yang1*, Jing Sun ${ }^{2}$ and Changsheng $\mathrm{Li}^{3}$ \\ ${ }^{1}$ Department of Anesthesiology and Perioperative Medicine, Zhengzhou University, China \\ ${ }^{2}$ Department of Psychiatry, Zhengzhou University, China \\ ${ }^{3}$ Department of Anesthesiology and Perioperative Medicine, Zhengzhou University, China
}

*Corresponding author: Chunyao Yang, Department of Anesthesiology, the Affiliated Cancer Hospital of Zhengzhou University, Zhengzhou, China.

Received Date: January 02, 2020

Published Date: January 17, 2020

Abstract

Objective: To explore the influence of maternal separation in early life on the neurodevelopment and young adult behavior of neonatal rats. Method Both male and female neonatal Sprague-Dawley rats were subjected to $2 \mathrm{~h}$ of maternal separation twice a day from postnatal days 2 (P2) through P9 followed by electroencephalogram (EEG) for $2 \mathrm{~h}$ on P10 or P11. Serum corticosteroid secretion and hippocampus expression of mGlu1R and mGlu5R were determined after EEG. Behavioral tests were examined by prepulse inhibition and elevated plus maze between P60 P70.

Results: The male group had increased corticosteroid level $(\mathrm{t}(10)=-5.458, \mathrm{P}<0.001)$ and decreased mGlu1R and mGlu5R expression in the hippocampi compared to the control group, with greater abnormity in EEG(This is defined by the total duration of seizure $(\mathrm{t}(10)=6.134, \mathrm{P}<0.001)$, number of episodes $(\mathrm{t}(10)=3.669, \mathrm{P}=0.016)$ and duration of a single episode $(\mathrm{t}(10)=2.916, \mathrm{P}=0.009))$ compared to female pups. The male rats were more severely disturbed than female rats in prepulse inhibition and performed worse in elevated plus maze treatment.

Conclusion: Maternal separation in early life induced significant abnormities in EEG and alterations in expression of hippocampus mGlu1R and mGlu5R with greater changes in the corticosteroid level in males and extended behavior abnormalities in young adult rats.

Keywords: Neurodevelopment; Electroencephalogram; Maternal separation; Behavior

\section{Introduction}

The number of young people from rural areas that go to work in cities is increasing every year in China. In order to reduce the life burden or work convenience, the young parents usually leave their kids in native family. The children living with their grandparents and other dependents are deprived parental care and love. Children in this special child growth environment may have an increased risk of developing mood and anxiety disorders. Clinical studies showed that exposure to early adverse events such as childhood sexual abuse and trauma are associated with an increased risk of developing psychiatric disorders in adulthood [14]. There is no data about whether the children deprived parent care have prolonged influence in adulthood; however, in animal experiments, hypothalamus corticotrophin releasing hormone (CRH) abnormality is emerging in the neonatal rats with only one 2-hour separation from their dams [5]. After repeated separation for 3 hours per day, rats showed a greater hypothalamic-pituitary- adrenal (HPA) axis response to acute restraint stress compared to non-separated control rats [6]. Before we found a brief exposure to ET combined with a subsequent episode of stress early in life induced significant alterations in expression of amygdala CRH and some gene expression in male rats [7]. Even $2 \mathrm{~h}$ maternal separation showed abnormal electroencephalogram activity [8].

The first two postnatal weeks is critical period, because a complex program of gene expression involved in structural and functional plasticity in neurodevelopment, forms that is responsible for subsequent brain functions and emotional behaviors during adulthood. Little is known about the ontogeny and function of the neuroendocrine system of children separated from their parents and its contribution to neurology abnormal development, growth failure, or depression after repeated maternal separation during the childhood. It has been suggested that neuronal activity modifies the chromatin complex at the crossroads of transcriptional activation 
and regulated genetic expression $[9,10]$. There is increasing evidence demonstrating that aberrant transcriptional regulation is one of the key components of the pathogenesis of several neuropsychiatric disorders, including mood disorders $[11,12]$. The mGlu1R and mGlu5R genes are essential for synaptic encoding of spatial experience and important mediators for a wide range of chronic psychosocial stress-induced alterations in humans [13,14]. The mGlu1 and mGlu5 receptors are involved in mechanisms of activitydependent synaptic plasticity and are targeted by drugs developed for the treatment of central nervous system (CNS) disorders [15]. In a previous study, we didn't find mGlu1 $\mathrm{R}$ and mGlu5R alternation in caudal brain of Shank3+/ $\Delta \mathrm{C}$ mice in previous study, but mGlu5R decreased in cultured rat hippocampal and cortical neurons in vitro study by Verpelli et al. $[16,17]$. The rat HPA axis is hypo-responsive in the first two postnatal weeks. The rat infant HPA shares many molecular and biological characteristics with the human HPA, so the rat maternal separation is an appropriate model to study the human neurodevelopment abnormalities.

The paradigm consisted of both male and female rats. The pups were separated from the dams for two 2-hour separation phases twice per day from postnatal day 2 through 9 to mimic the leftover kids neglected by their working parents. We aimed to examine the neurodevelopment vulnerability of deprived parentcare in childhood to behavioral abnormality in adulthood. We first clarified the neurological mechanism underlying the development of stress vulnerability in early maternally separated rats. We then investigated the sex difference in the neuroendocrine system and behavioral consequences of exposure to repeated maternal separation in infants.

\section{Methods and Materials}

\section{Animals}

All experimental procedures were approved by the Institutional Animal Care and Use Committee of the Affiliated Cancer Hospital of Zhengzhou University. Male and female Sprague-Dawley rats were studied. Animals were housed under controlled illumination (12-h light/dark, lights on at 7:00 a.m.) and temperature $\left(23-24^{\circ} \mathrm{C}\right)$ with free access to food and water. Within $24 \mathrm{~h}$ of delivery, litters were culled to 12 pups. At the beginning of each experiment the pups were determined to be well nourished as judged by their stomachs being full of milk (detectable through the transparent abdominal wall). Delivery was verified at 12-hour intervals, and the day of birth was considered postnatal day 0 . On postnatal day 2, pups were placed with mothers in cages and maternal separation treatment began. The data reported in this study was collected from 120 rats distributed evenly based on sex.

\section{Maternal separation procedure}

Twelve experimental groups were studied. On postnatal day 2 pups in experimental litters were separated from their dams twice daily at the Same time each day (9:00a.m.-11:00a.m. and 15:00p.m.17:00p.m.) in the morning and afternoon. Each subsequent day through P9 to mimic the children separated from their parents.
Maternal separated pups were kept in a temperature-controlled chamber $\left( \pm 37^{\circ} \mathrm{C}\right)$ with a continuous supply of oxygen $(1.5 \mathrm{~L} /$ min). The control animals were raised undisturbed. After the last separation pups were returned to their respective dams. Rats were weaned on P21 and group-housed thereafter with same-sex littermates. On P60, a subset of rats were subjected to behavioral tests (Figure 1, A).

\section{Electroencephalogram recording}

After sequential maternal separation, electroencephalogram (EEG) recordings were performed on P10 or P11 in a thermostated chamber $\left( \pm 37^{\circ} \mathrm{C}\right)$ with a continuous supply of oxygen $(1.5 \mathrm{~L} / \mathrm{min})$ as previous described8. Gas was monitored by a calibrated Datex side stream analyzer (Datex-Ohmeda, Helsinki, Finland), which sampled from the interior of the animal chamber. A minor operation was performed under isoflurane anesthesia (1.6-2.0\%) by the same colleague, where four screw electrodes were implanted bilaterally in the occipital and frontal regions of the rat pup skull with the left frontal electrode serving as the reference electrode. The electrode headmount was fixed by the screws and assured a good contact with the skull. After the operation, the rat moved to the EEG recording system. Continuous 2 hours EEG recordings were performed using an EEG/EMG system (Pinnacle Technology, Lawrence, KS) (Figure 1, A). Acquisition of the EEG was performed using the Sirenia software (Pinnacle Technology). The sampling interval per signal was $200 \mu \mathrm{s}$. Data was filtered offline using a bandpass Bessel (8pole) $0.04-56-\mathrm{Hz}$ filter. Sirenia CGMS analysis program assisted for the EEG data analysis. Electroencephalogram patterns that were characterized by the amplitude at least three times higher than baseline and abruptly reverted to baseline were defined as seizure electroencephalogram patterns. In most cases these patterns start as high frequency-low amplitude activity that developed to increased amplitude and decreased frequency and then abruptly reverted to baseline activity. The total duration, number of episodes and average duration of episode were calculated. The investigators analyzing the EEGs were blinded to the experimental conditions and all EEGs were reviewed by three independent reviewers.

\section{Corticosterone and hippocampus mGlu1R and mGlu5R determination}

For measurements of the corticosteroid secretion, blood samples $(\sim 300 \mu \mathrm{L})$ were collected using the "tail clip" method after the EEG recoding. Corticosterone concentration was determined using a commercial ELISA kit (Cayman Chemical Company, Ann Arbor, MI) following the manufacturer's instructions. When blood collecting finished, the rats were decapitated and the hippocampi were quickly dissected from the brain and frozen in liquid nitrogen. The mGlu1R and mGlu5R protein in the hippocampus were detected as previously described.16 Briefly, after the tissues were homogenized and the protein was extracted, mGlu1R and mGlu5R were resolved by sodium dodecyl sulfate-polyacrylamide gel electrophoresis and electrotransferred to nitrocellulose membranes. The membranes were blocked in $0.1 \%$ Tween-20 in Tris-HCl-buffered saline (TBST) containing 5\% nonfat milk for 
$1 \mathrm{~h}$ at room temperature and then immunoblotted with primary antibodies(anti-mGlu1R: 1:1000, Cell-signaling, Danvers, MA; antimGlu5R: 1:5000, $\beta$-actin:1:100,000, Sigma-Aldrich, St. Louis, MO) in TBST buffer containing $5 \%$ nonfat milk overnight at $4^{\circ} \mathrm{C}$. After being washed extensively in TBST, the membranes were incubated for $1 \mathrm{~h}$ with horseradish peroxidase-conjugated anti-mouse or anti-rabbit immunoglobulin (Bio-Rad Laboratories, Hercules, CA) at a dilution of 1:2,000. Proteins were detected by enhanced chemiluminescence (Amersham, Piscataway, NJ). $\beta$-actin served as a loading control. The immunoblotting bands were quantified by densitometry using Image J software (National Institutes of Health, Bethesda, MD) and analyzed.

\section{Behavioral tests}

Young adult rats were sequentially evaluated in the elevated plus maze (EPM) and prepulse inhibition (PPI) of the acoustic startle response at $\mathrm{P} 60 \sim \mathrm{P} 70$.

Assessment of behavior in the EPM: The elevated plus maze is a commonly used test to measure anxiety in rodents and consists of two open arms and two closed arms that are elevated above the ground. Rats that are less anxious will spend more time in the open arms compared to control rats, and rats that are more anxious will spend less time in the open arms compared to controls. This test provides a sensitive measure of anxiety, and we used the test to evaluate whether seizures on postnatal days 10-11 result in anxiety. The EPM studies were performed using the EPM apparatus and BIO-EPM 3C video tracking software (EB Instruments, Pinellas Park, FL) during the light phase of the dark-light cycle. The maze consists of two opposing open $(50 \times 10 \times 0.5 \mathrm{~cm})$ and two enclosed $(50 \times 10 \times 45 \mathrm{~cm})$ arms elevated $75 \mathrm{~cm}$ above the floor, with a $0.5-\mathrm{cm}$ edge on the open arms. Animals were placed in the center square facing an open arm and allowed to acclimate to the maze for $5 \mathrm{~min}$, at which time they were removed from the apparatus. During EPM testing, each rat's behavior was recorded using BIO-EPM 3C video tracking software. The percentage of time spent in the open and enclosed arms, and the total distance traveled during $5 \mathrm{~min}$ of recording as an index of locomotor activity were compared.

Measurements of the acoustic startle response and PPI of startle: The PPI of startle tests were performed using the SR-Lab startle apparatus (San Diego Instruments, San Diego, CA) to assess sensorimotor gating. Testing occurred during the light phase of the dark light cycle. At the beginning of every testing session, each animal was placed in the cylindrical animal enclosure and was then exposed to a $75 \mathrm{~dB}$ white noise background for $5 \mathrm{~min}$ acclimation period. The acclimation period was followed by a test session that consisted in of five different trials: a $120 \mathrm{~dB} 40 \mathrm{~ms}$ pulse only; a $120 \mathrm{~dB} 40 \mathrm{~ms}$ pulse preceded by a prepulse of a $20 \mathrm{~ms}$ duration at 5, 10, and $15 \mathrm{~dB}$ above background; and a no-stimulus tri of background noise. The delay between the onset of the prepulse and the onset of the pulse was $100 \mathrm{~ms}$. The trials were presented in pseudorandom order with variable inter-trial intervals averaging $15 \mathrm{~s}$. The first four trials and last three trials consisted of $120 \mathrm{~dB}$ pulse only trials. All five types of trials were presented eight times, each in pseudorandom order after the first four and before the last three pulse-only trials. The \%PPI for each prepulse level was calculated using the following formula: \%PPI =100x [(pulse alone) - (prepulse + pulse)]/pulse alone [18].

\section{Statistical Analysis}

Values are reported as mean \pm SEM. SigmaPlot 12.5 software (Systat Software, Inc., Point Richmond, CA) was used for statistical analyses. Single comparisons were tested using the t-test. All comparisons were run as two-tailed tests. A P $<0.05$ was considered significant.

\section{Results}

The data clearly show that males appear to be more responsive to maternal separation compared to females. Maternal separation causes more seizures events in electroencephalograms of male than female P10/P11 rat pups. As explained in detail in experimental procedures, we separated the pups and dams from P2 through P9 and test the gender difference of electroencephalograms in P10 or P11. Analysis of 2 hour electroencephalographic recordings revealed statistically significant differences in seizure activities in male and female rat pups. The total duration of seizure $(\mathrm{t}(10)=6.134$, $\mathrm{P}<0.001)$, the number of episodes $(\mathrm{t}(10)=3.669, \mathrm{P}=0.016)$ and the duration of a single episode $(\mathrm{t}(10)=2.916, \mathrm{P}=0.009)$ during 2 hours were higher in male $(n=10)$ than female $(n=10)$ rat pups. The electroencephalographic recording was normal in control pups with no seizure activity discovered (Figure2).

Male pups showed higher vulnerability in response to maternal separation stress than female pups.

Stressors activate the hypothalamic-pituitary-adrenal (HPA) axis, triggering secretion and compensatory synthesis of hypothalamic corticotropin-releasing hormone(CRH). CRH is located in peptidergic neurons in the paraventricular hypothalamic nucleus, is secreted from nerve terminals to influence rapid hormonal secretion from corticotrophs in the anterior pituitary $[19,20]$. In basal condition, male pups have greater plasma corticosterone levels than female pups. Maternal deprivation increased CORT levels in male in comparison to the control pups $(\mathrm{t}(10)=-5.458, \mathrm{P}<0.001)$. However, the female pups didn't showed hyper reactivity of the HPA axis to repeated separation stress $(\mathrm{t}(10)=-0.174, \mathrm{P}=0.864)$. After the EEG experiments, the CORT level was higher in male pups compared to female pups $(\mathrm{t}(10)=9.245, \mathrm{P}<0.001)$ (Figure1, D).

Among all glutamatergic system components, metabotropic receptors play a main role in regulating neuronal excitability and synaptic plasticity. mGlu1R and mGlu5R present in the hippocampus are important in regulating the activity of the HPA axis after stress. mGlu1R and mGlu5R expression decrease significantly in male pups deprived mother care in comparison to the control (Figure1, B).

Maternal separation in the neonatal period induced extended behavior abnormalities in adulthood. The long-term effects of maternal separation were evaluated by assessing sensorimotor gating. Both male $(\mathrm{t}(10)=-15.4, \mathrm{P}<0.001)$ and female $(\mathrm{t}(10)=-6.583$, 
$\mathrm{P}<0.001$ ) rats with neonatal maternal separation from $\mathrm{P} 2$ to $\mathrm{P} 9$ exhibited reduced PPI of the acoustic startle response at lowest prepulse intensity $(5 \mathrm{~dB})$ when compared to rats in the control. But, male rats showed reduced PPI responses at prepulse intensities of $10 \mathrm{~dB}$ compared to control group $(\mathrm{t}(10)=-10.900, \mathrm{P}<0.001)$. PPI responses at prepulse intensities of $15 \mathrm{~dB}$ were similar across all groups and between sexes (Figure1, E).
In the elevated plus maze, adult female rats traveled similar total distances and stayed in open arms for similar times in comparison to the control, but male rats spent a shorter time in open $\operatorname{arms}(\mathrm{t}(10)=-4.871, \mathrm{P}<0.001)$ of the EPM and covered shorter distances when compared to the control $(\mathrm{t}(10)=-7.928, \mathrm{P}<0.001)$ (Figure1, C1\&C2).

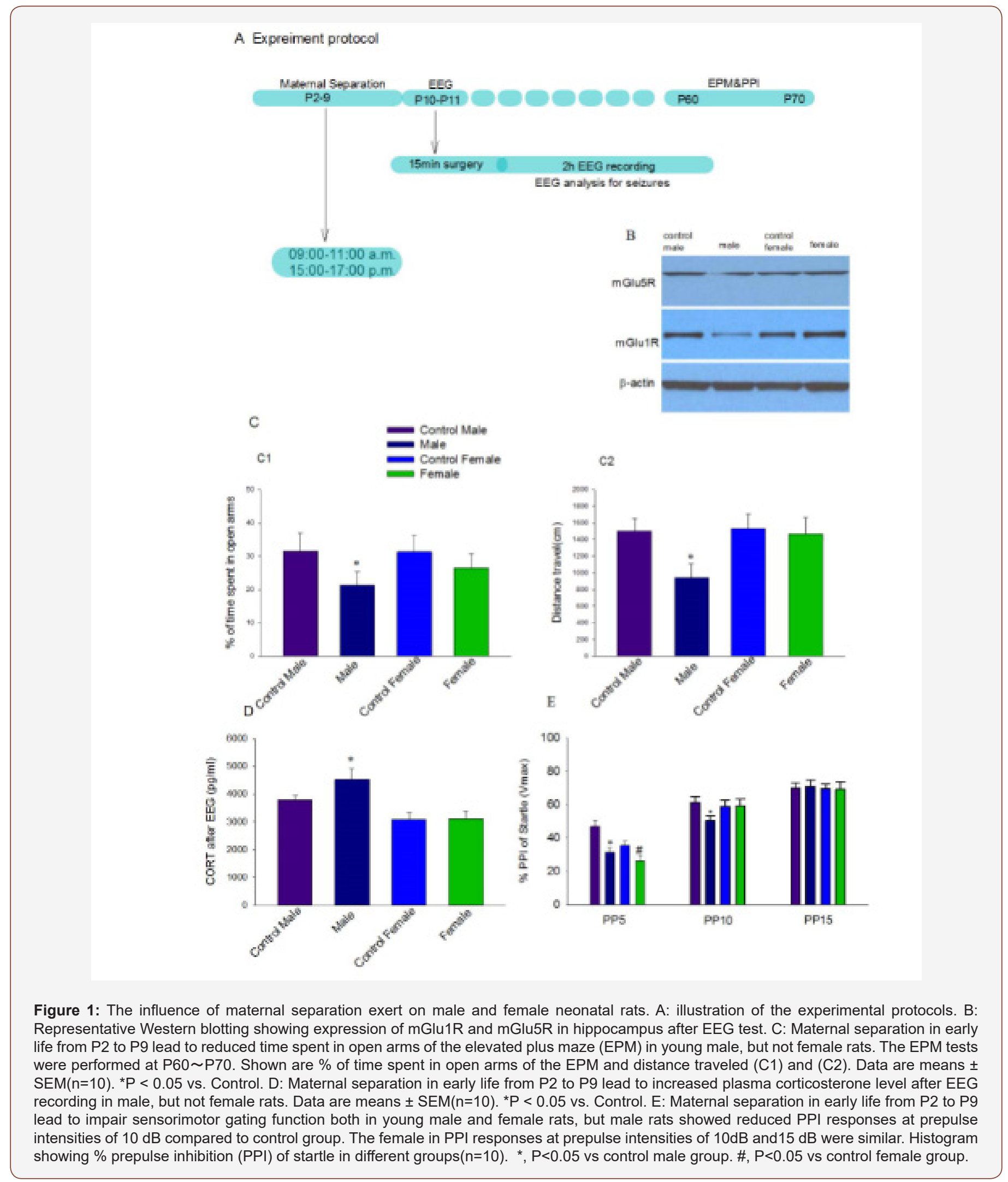




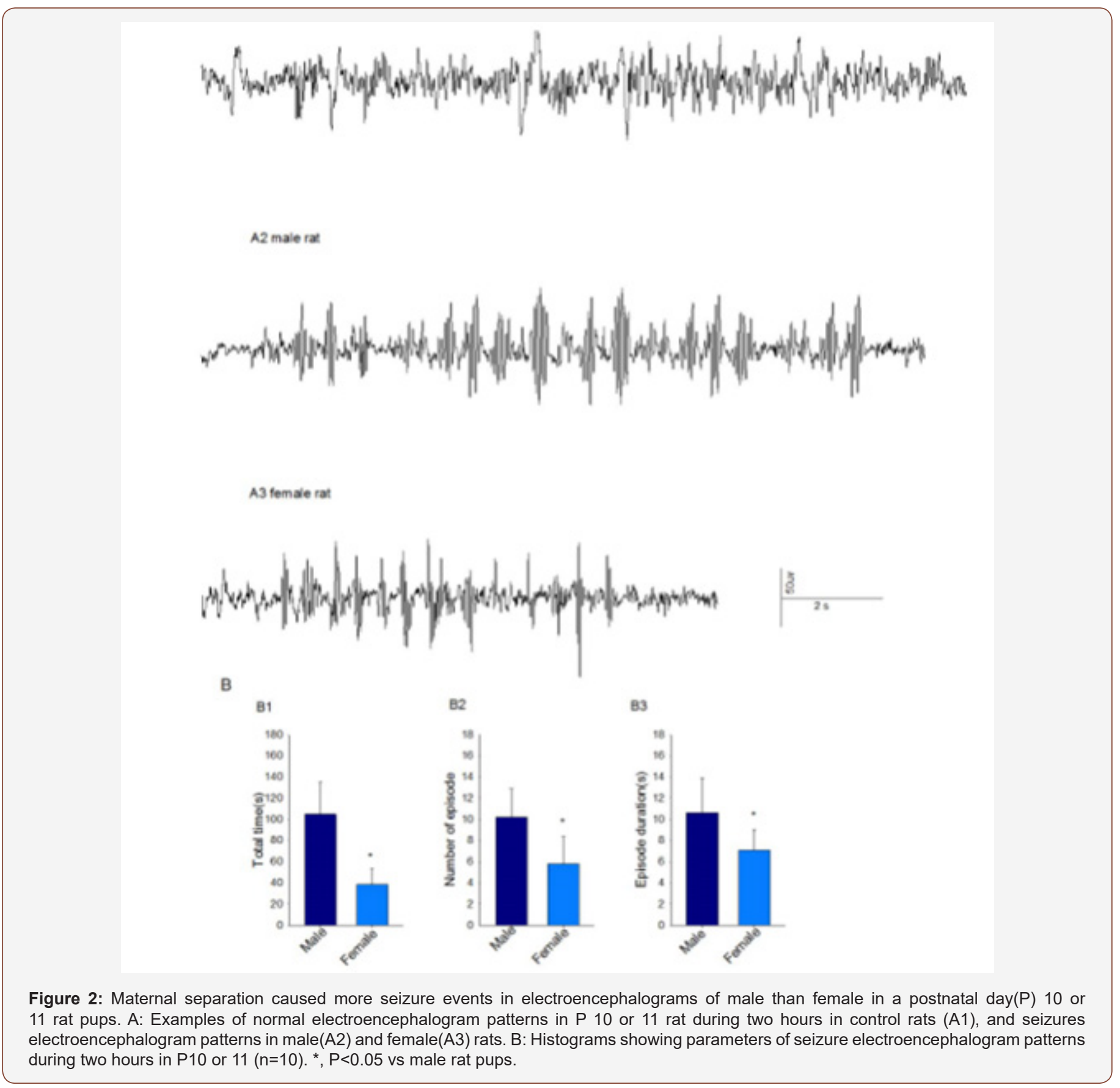

\section{Discussion}

In the present study, we found that maternal separation exerted a vulnerable stress on male rats compared to their counterpart females. At the molecular level, maternal separation decreased the expression of mGlu1R and mGlu5R, and increased the corticosterone levels. Prolonged maternal separation during early infancy led to disturbances in prepulse inhibition and behavioral detriments in young adults especially for males. Early life chronic stress is one of the most prominent environmental factors associated with an increased risk of developing mood and anxiety disorders [21]. Early separation may act as a stressor and have adverse effects on adult neural and behavioral outcomes [7,22]. Maternal separation is an animal model of early life stress that modulates the HPA axis and studies the neurobiological underpinnings of disruption of the mother-infant relationship and loss of parental care, a highly prevalent condition in humans [23]. This paradigm may more closely approximate the human situation of children separated from their parents who are deprived parental care in infancy. The human brain requires a wide variety of experiences and environmental inputs in order to develop normally. Children who are neglected by caregivers or raised in institutional environments are deprived of numerous types of species-expectant environmental experiences that confer risk for internalizing and externalizing psychopathology [24]. Neglect disrupts the development of brain, exaggerating the activity of the HPA axis and correlating to adult depression and anxiety [25]. Depression, anxiety, and poor growth are well established consequences of chronic neglect occurring in infancy. These abnormal attachments are, in part, correlated with disregulation of the HPA axis, as shown by elevated corticosterone 
levels in separated animals. It should be noted that daily separation from dams in neonatal exerted an important impression on males. This study showed that mGlu1R and mGlu5R expression were decreased in male pups compared to female pups, but corticosterone levels were increased significantly in male pups. mGlu1R and mGlu5R present in the hippocampus are important in regulating the activity of the HPA axis after stress and within the hypothalamus to regulate rapid hormonal responses to stress [26]. Glutamate is essential for learning and memory processing, specifically mGlu1R and mGlu5R enhance NMDA receptors excitability and play a key role in neurodevelopment positive. Other animal experiments indicated that modulation of mGlu1 and mGlu5 receptors prevented and reversed ethanol-induced memory impairment [27-29]. The interaction between glutamate and NMDA receptors is vital for synaptic plasticity and cognition. Stimulated glutamate receptors enhance NMDAR responses in the hippocampus leading to gene expression, synaptic plasticity, and memory consolidation [30]. Cognitive dysfunction may be improved by targeting mGlu5R with an appropriate therapy [31]. mGlu1R and mGlu5R alterations can lead to molecular imbalance between the excitation and inhibition underlying the emergence of a schizophrenic-like phenotype [32]. It is important to evaluate the potential of glutamate modulators in reversing the deficits characterizing the schizophrenic pathology. Together, these findings suggest that decreased mGlu1R and mGlu5R might be associated with increased HPA axis response to stress. This increased exposure to corticosterone and hyper-reactivity of the HPA axis can increase depression and anxiety-related behaviors in rodents. Among all glutamatergic system components, metabotropic receptors play a main role in regulating neuronal excitability and synaptic plasticity, specifically, mGlu 1 and mGlu5 play important role in environmental modulation of schizophrenia-related behavioral impairments [33]. There is no known causal relationship between epilepsy and mGlu1R and mGlu5R; however, the male pups showed higher EEG abnormalities that are characteristic of seizure activities. Behaviors tested in adults suggest that they may share anatomical and molecular mechanisms, especially during brain development. A single 24-hour period of maternal deprivation at postnatal day 9 led to disturbances in a pup's prepulse inhibition and latent inhibition [34]. In the PPI test, male adult rats showed disturbance in the startle response at both $5 \mathrm{~dB}$ and $10 \mathrm{~dB}$, but female rats only suffered $5 \mathrm{~dB}$ impairment on PPI of startle. In the EPM treatment, female rats spent more time in the open arm and travelled longer distance. While male rats moved anxiously around the open field seeking escape routes or an area of shelter, female rats seemed more bold and wise. Since spatial learning requires mGlu1R and mGlu5R signaling in the dorsal hippocampus [35], the male rats anxiety might be related to the down-regulated expression of mGlu1R and mGlu5R caused by maternal separation.

The neurodevelopment showed sex-specific relations to the aberrations in the HPA axis and neurological gene expression, suggesting that maternal separation could contribute to decreased hippocampal neurogenesis and altered neuroendocrine axis
[36]. In summary, our study confirmed that maternal separation during early life is a chronic stress that could lead to behavior and neurochemical abnormalities in rats, and this model highlights the special growth environment that is influential in young adult human development

\section{Conclusion}

Maternal separation in early life induced significant abnormities in EEG and alterations in expression of hippocampus mGlu1R and mGlu5R with greater changes in the corticosteroid level in males and extended behavior abnormalities in young adult rats.

\section{Acknowledgement}

This work was supported by the Department of Anesthesiology and Perioperative Medicine, the Affiliated Cancer Hospital of Zhengzhou University, Henan cancer hospital, Henan, China. The colleagues gave meaningful advice and help in the experiment. This study was support by the National Natural Science Foundation of China.

\section{Conflict of Interest}

There is no conflict of interest in the study and the preliminary data was unpublished.

\section{References}

1. Choi KW, Batchelder AW, Ehlinger PP, Safren SA, O'Cleirigh C (2017) Applying network analysis to psychological comorbidity and health behavior: Depression, PTSD, and sexual risk in sexual minority men with trauma histories. J Consult Clin Psychol 85(12): 1158-1170.

2. Williams LM, Debattista C, Duchemin AM, Schatzberg AF, Nemeroff CB (2016) Childhood trauma predicts antidepressant response in adults with major depression: data from the randomized international study to predict optimized treatment for depression. Transl Psychiatry 6: e799.

3. Ballard ED, Van Eck K, Musci RJ, Hart SR, Storr CL, et al. (2015) Latent classes of childhood trauma exposure predict the development of behavioral health outcomes in adolescence and young adulthood. Psychol Med 45(15): 3305-3316.

4. Kavanaugh BC, Dupont-Frechette JA, Jerskey BA, Holler KA (2017) Neurocognitive deficits in children and adolescents following maltreatment: Neurodevelopmental consequences and neuropsychological implications of traumatic stress. Applied neuropsychology Child 6: 64-78.

5. Ju LS, Yang JJ, Gravenstein N, Seubert CN, Morey TE, et al. (2017) Role of environmental stressors in determining the developmental outcome of neonatal anesthesia. Psychoneuroendocrinology 81: 96-104.

6. Uchida S, Hara K, Kobayashi A, Funato H, Hobara T, et al. (2010) Early life stress enhances behavioral vulnerability to stress through the activation of REST4-mediated gene transcription in the medial prefrontal cortex of rodents. The Journal of neuroscience: the official journal of the Society for Neuroscience 30: 15007-150018.

7. Yang J, Lingsha J, Yang C, Xue J, Setlow B, et al. (2018) Effects of combined brief etomidate anesthesia and postnatal stress on amygdala expression of Cl- cotransporters and corticotropin-releasing hormone and alcohol intake in adult rats. Neuroscience letters.

8. Yang C, Li C, Sun J, Lu X (2019) Role of estradiol in mediation of etomidate-caused seizure-like activity in neonatal rats. International journal of developmental neuroscience: the official. Journal of the International Society for Developmental Neuroscience 78: 170-177.

9. Oey NE, Leung HW, Ezhilarasan R, Zhou L, Beuerman RW, et al. (2015) A Neuronal Activity-Dependent Dual Function Chromatin-Modifying Complex Regulates Arc Expression. eNeuro 2. 
10. Flavell SW, Greenberg ME (2008) Signaling mechanisms linking neuronal activity to gene expression and plasticity of the nervous system. Annual review of neuroscience 31: 563-590.

11. Uchida S, Hara K, Kobayashi A, Fujimoto M, Otsuki K, et al. (2011) Impaired hippocampal spinogenesis and neurogenesis and altered affective behavior in mice lacking heat shock factor 1 . Proceedings of the National Academy of Sciences of the United States of America 108: 1681-1186.

12. Higuchi F, Uchida S, Yamagata H, Otsuki K, Hobara T, et al. (2011) State-dependent changes in the expression of DNA methyltransferases in mood disorder patients. Journal of psychiatric research 45: 12951300.

13. Peterlik D, Stangl C, Bauer A, Bludau A, Keller J, et al. (2017) Blocking metabotropic glutamate receptor subtype 5 relieves maladaptive chronic stress consequences. Brain, behavior, and immunity 59: 79-92.

14. Dietz B, Manahan-Vaughan D (2017) Hippocampal long-term depression is facilitated by the acquisition and updating of memory of spatial auditory content and requires mGlu5 activation. Neuropharmacology 115: 30-41.

15. Di Menna L, Joffe ME, Iacovelli L, Orlando R, Lindsley CW, et al. (2018) Functional partnership between mGlu3 and mGlu5 metabotropic glutamate receptors in the central nervous system. Neuropharmacology 128 : 301-313.

16. Li C, Schaefer M, Gray C, Yang Y, Furmanski O, et al. (2017) Sensitivity to isoflurane anesthesia increases in autism spectrum disorder Shank3(+/c) mutant mouse model. Neurotoxicology and teratology 60: 69-74.

17. Verpelli C, Dvoretskova E, Vicidomini C, Rossi F, Chiappalone M, et al. (2011) Importance of Shank3 protein in regulating metabotropic glutamate receptor 5 (mGluR5) expression and signaling at synapses. The Journal of biological chemistry 286: 34839-34850.

18. Geyer MA, Dulawa SC (2003) Assessment of murine startle reactivity, prepulse inhibition, and habituation. Current protocols in neuroscience Chapter 8: Unit 8. 17.

19. Rivier J, Spiess J, Vale W (1983) Characterization of rat hypothalamic corticotropin-releasing factor. Proceedings of the National Academy of Sciences of the United States of America 80: 4851-4855.

20. Yi SJ, Baram TZ (1994) Corticotropin-releasing hormone mediates the response to cold stress in the neonatal rat without compensatory enhancement of the peptide's gene expression. Endocrinology 135: 23642368.

21. Godoy LD, Umeoka EHL, Ribeiro DE, Santos VR1, Antunes-Rodrigues J, et al. (2018) Multimodal early-life stress induces biological changes associated to psychopathologies. Hormones and behavior 100: 69-80.

22. Morris MJ, Beilharz JE, Maniam J, Reichelt AC, Westbrook RF (2015) Why is obesity such a problem in the 21st century? The intersection of palatable food, cues and reward pathways, stress, and cognition. Neuroscience and biobehavioral reviews 58: 36-45.

23. Suchecki D (2018) Maternal regulation of the infant's hypothalamic-pituitary-adrenal axis stress response: Seymour 'Gig' Levine's legacy to neuroendocrinology. Journal of neuroendocrinology 30: e12610.
24. McLaughlin KA, Sheridan MA, Nelson CA (2017) Neglect as a Violation of Species-Expectant Experience: Neurodevelopmental Consequences. Biological psychiatry 82: 462-471.

25. Rosenfeld P, Wetmore JB, Levine S (1992) Effects of repeated maternal separations on the adrenocortical response to stress of preweanling rats. Physiology \& behavior 52: 787-791.

26. Baram TZ, Hatalski CG (1998) Neuropeptide-mediated excitability: a key triggering mechanism for seizure generation in the developing brain. Trends in neurosciences 21(11): 471-476.

27. O’Riordan KJ, Hu NW, Rowan MJ (2018) Physiological activation of mGlu5 receptors supports the ion channel function of NMDA receptors in hippocampal LTD induction in vivo. Scientific reports 8: 4391.

28. Cavalier M, Ben Sedrine A, Thevenet L, Crouzin N, Guiramand J, et al. (2018) Disturbance of Metabotropic Glutamate Receptor-Mediated Long-Term Depression (mGlu-LTD) of Excitatory Synaptic Transmission in the Rat Hippocampus After Prenatal Immune Challenge. Neurochemical research 44(3): 609-616.

29. Marszalek-Grabska M, Gibula-Bruzda E, Bodzon-Kulakowska A, Suder P, Gawel K, et al. (2018) Effects of the Positive Allosteric Modulator of Metabotropic Glutamate Receptor 5, VU-29, on Impairment of Novel Object Recognition Induced by Acute Ethanol and Ethanol Withdrawal in Rats. Neurotoxicity research 33: 607-620.

30. Krania P, Dimou E, Bantouna M, Kouvaros S, Tsiamaki E, et al. (2018) Adenosine A2A receptors are required for glutamate mGluR5- and dopamine D1 receptor-evoked ERK1/2 phosphorylation in rat hippocampus: involvement of NMDA receptor. Journal of neurochemistry 145: 217 231.

31. Nardecchia F, Orlando R, Iacovelli L, Colamartino M, Fiori E, et al. (2018) Targeting mGlu5 Metabotropic Glutamate Receptors in the Treatment of Cognitive Dysfunction in a Mouse Model of Phenylketonuria. Frontiers in neuroscience 12: 154 .

32. Luoni A, Gass P, Brambilla P, Ruggeri M, Riva MA, et al. (2018) Altered expression of schizophrenia-related genes in mice lacking mGlu5 receptors. European archives of psychiatry and clinical neuroscience 268 : 77-87.

33. Burrows EL, McOmish CE, Buret LS, Van den Buuse M, Hannan AJ (2015) Environmental Enrichment Ameliorates Behavioral Impairments Modeling Schizophrenia in Mice Lacking Metabotropic Glutamate Receptor 5. Neuropsychopharmacology: official publication of the American College of Neuropsychopharmacology 40: 1947-1956.

34. Aksic M, Radonjic NV, Aleksic D, Jevtić G, Marković B, et al. (2014) Longterm effects of maternal deprivation on the neuronal soma area in the rat neocortex. BioMed research international: 235238.

35. Tan SZ, Ganella DE, Dick AL, Duncan JR, Ong-Palsson E, et al. (2015) Spatial Learning Requires mGlu5 Signalling in the Dorsal Hippocampus. Neurochemical research 40: 1303-1310.

36. Roque A, Ochoa-Zarzosa A, Torner L (2016) Maternal separation activates microglial cells and induces an inflammatory response in the hippocampus of male rat pups, independently of hypothalamic and peripheral cytokine levels. Brain, behavior, and immunity 55: 39-48. 